\title{
The geometry effect on transverse oscillations of coronal loops (Research Note)
}

\author{
M. V. Dymova and M. S. Ruderman
}

\begin{abstract}
Department of Applied Mathematics, University of Sheffield, Hicks Building, Hounsfield Road, Sheffield S3 7RH, UK e-mail: M.S.Ruderman@sheffield.ac.uk
\end{abstract}

Received 29 June 2006 / Accepted 7 August 2006

\section{ABSTRACT}

\begin{abstract}
In the recent study of nine transversely oscillating coronal loops Verwichte et al. (2004) found that in two events the fundamental mode and the first overtone were observed simultaneously, and the ratio of their periods was different from two predicted by the uniform tube model. This observation inspired Andries et al. (2005a, ApJ, 624, L57) to use this ratio for determining the atmospheric scale height. When doing so these authors assumed that the loop has a semicircular shape. In our paper we address the question how strongly the method suggested by Andries et al. (2005a) depends on the assumed loop shape. We found that this dependence is fairly strong, so that observations of frequencies of the fundamental mode and first overtone have to be supplemented with observations of the loop shape.
\end{abstract}

Key words. magnetohydrodynamics (MHD) - plasma - waves - Sun: oscillation - Sun: corona

\section{Introduction}

Recent space based observations of transverse coronal loop oscillations, first reported by Aschwanden et al. (1999) and Nakariakov et al. (1999), prompted various theoretical studies on the subject and gave a new boost to coronal seismology approach first suggested by Uchida (1970) and Roberts et al. (1984). Solar coronal seismology is basically a comparison of the wave properties obtained from the observations with those derived from theoretical investigation to produce seismic information on the physical parameters in the solar corona. Nakariakov et al. (1999) first suggested to model the observed standing transverse coronal loop oscillations as fast kink modes of magnetic flux tubes. This approach has been widely used since then. As far as periods are concerned, the uniform tube model gives a good agreement with observations, but appears to be unable to provide information on the damping rates since it does not take the radial density variation into account. To explain the rapid damping of coronal loop oscillations Ruderman \& Roberts (2002) solved the initial value problem for a tube with the radially dependent density and obtained the expression for the damping rate due to resonant absorption. Observations by Schrijver et al. (2002) revealed that oscillations occur in loops with heights comparable with the density scale height in the corona, which suggests that the stratification is significant. Andries et al. (2005b) and Arregui et al. (2005) took this effect into account in the numerical study of the fast kink mode of transverse oscillations of a magnetic tube with the equilibrium density varying both in the radial and longitudinal direction. Dymova \& Ruderman (2006) solved the same problem analytically. They found that the damping rate does not depend on the stratification and is given by the same expression as in the unstratified case. If radial density variation is confined to a thin layer at the tube boundary, it only causes a weak resonant damping rate and a small and thus negligible shift of the oscillation frequency. To single out the effect of longitudinal density stratification on the oscillation frequencies we assume in the present study that the density is a function of the longitudinal coordinate only.

When analyzing 9 cases of standing transverse coronal loop oscillations observed by TRACE Verwichte et al. (2004) found multiple harmonic oscillations. In two loops they detected two different frequencies and interpreted them as the fundamental mode, with the amplitude maximum at the apex, and the first overtone with a node at the apex. An important feature of these oscillations is that the ratios of the periods of the fundamental mode and the first overtone, $P_{\mathrm{f}} / P_{\mathrm{o}}=1.81 \pm 0.25,1.64 \pm$ 0.23 , for these two loops are clearly different from the theoretically obtained value of 2 for the uniform tube model. Andries et al. (2005a) suggested that this deviation of $P_{\mathrm{f}} / P_{\mathrm{o}}$ from 2 can be an efficient seismological tool for obtaining information on the density scale height in the corona. They considered a magnetic flux tube of a semi-circular shape embedded in an isothermal atmosphere, and numerically calculated the dependence of $P_{\mathrm{f}} / P_{\mathrm{o}}$ on the atmospheric scale height. Then they used this dependence to determine the atmospheric scale height in the two cases of multiple harmonic loop oscillations reported by Verwichte et al. (2004). As a result, Andries et al. (2005a) obtained quite reasonable values for the atmospheric scale height, $65 \mathrm{Mm}$ and $36 \mathrm{Mm}$. Unfortunately, the lack of precision in observational data leads to significant error bars for these computed scale heights, even allowing negative scale heights.

It was also pointed out by Andries et al. (2005a) that the longitudinal density stratification might not be the only cause for the deviation of $P_{\mathrm{f}} / P_{\mathrm{o}}$ from the uniform value of 2 . They wrote that "non-circular curvature of the loop, variable cross section, etc., could influence the ratio of the frequencies as well". The main purpose of the present paper is to investigate further the possibility of calculating coronal density scale height from the observed parameters of oscillating loops. In this study we aim to analyse the dependence of $P_{\mathrm{f}} / P_{\mathrm{o}}$ on the density stratification parameter, stratification profile and, in particular, the geometry of the loop. We use the method developed by Dymova \& Ruderman (2005) to calculate the ratio $P_{\mathrm{f}} / P_{\mathrm{o}}$ in a thin longitudinally stratified loop 


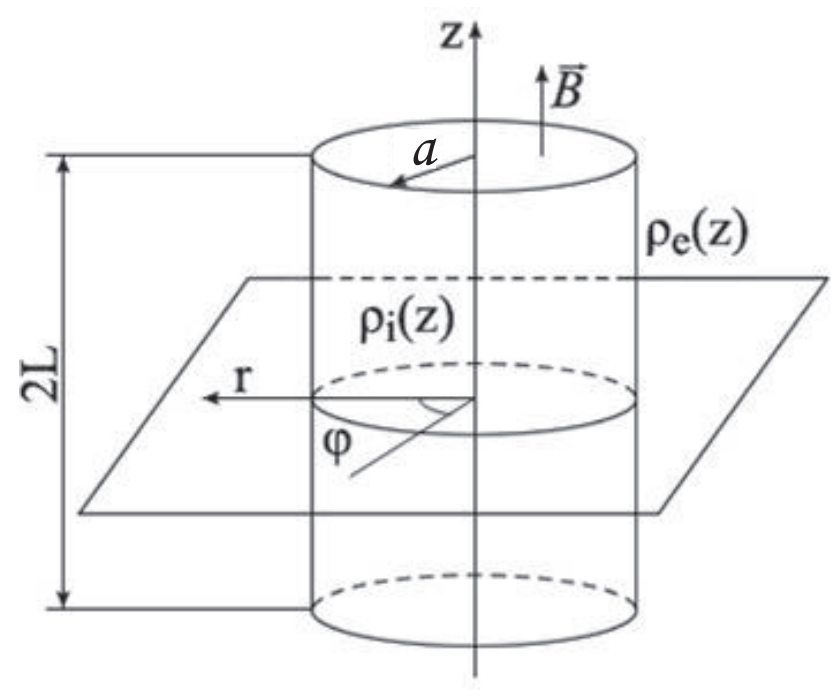

Fig. 1. The sketch of the equilibrium state. The ends of the magnetic tube are assumed to be frozen in a dense photospheric plasma.

with a sharp boundary. Since Dymova \& Ruderman (2005) approach allows arbitrary longitudinal density profile we choose it dependent on the loop shape.

\section{Analysis}

We use the method for calculating the kink MHD mode of loop oscillations developed by Dymova \& Ruderman (2005). The equilibrium configuration (Fig. 1) is a straight cylindrical flux tube of radius $a$ and length $2 L$ with constant magnetic field $B$ directed along the tube axis. We take the densities inside the tube, $\rho_{\mathrm{i}}(z)$, and in the surrounding coronal medium, $\rho_{\mathrm{e}}(z)$, to be functions of longitudinal coordinate $z$ only. We also adopt the assumption of uniform stratification, i.e. $\frac{\rho_{\mathrm{e}}(z)}{\rho_{\mathrm{i}}(z)}=\chi<1$ is constant.

Here we would like to make two comments on the equilibrium configuration considered in this paper. The first comment concerns the shape of coronal magnetic loops. The real coronal loops are curved magnetic flux tubes. In our papers we use a straight magnetic tube with the same variation of density along the tube as in a curved coronal loop. This simplification is only acceptable if the "straightening" of a loop does not affect very much the eigenfrequencies of its transverse oscillations. Van Doorsselaere et al. (2004) studied analytically the transverse oscillations of a thin magnetic tube of semi-circular shape with the density varying only in the direction perpendicular to the tube axis. They found that the difference between the eigenfrequencies of transverse oscillations of the straight and semi-circular tubes is of the order $\epsilon^{2}=(a / L)^{2}$. We do not see any reason why this estimate should change when the tube is stratified, i.e. when the density varies along the tube as well. Since for real coronal loops $\epsilon^{2} \lesssim 10^{-3}$, we believe that the loop "straightening" practically does not change the eigenfrequencies of its transverse oscillations.

Our second comment concerns the radial dependence of the density. In general, we cannot choose the magnetic configuration and the density distribution arbitrarily. Rather we have to calculate them by solving the magnetostatic equation determining the equilibrium in the presence of gravity,

$\nabla p-\frac{1}{\mu_{0}}(\nabla \times \boldsymbol{B}) \times \boldsymbol{B}=\boldsymbol{g} \rho$, where $p, \boldsymbol{B}$ and $\rho$ are the equilibrium pressure, magnetic field and density respectively, $\boldsymbol{g}$ is the gravity acceleration, and $\mu_{0}$ is the magnetic permeability of free space. The projection of this equation on the magnetic field direction yields

$\boldsymbol{b} \cdot \nabla p=g_{B} \rho$,

where $\boldsymbol{b}=\boldsymbol{B} / \boldsymbol{B}$ is the unit vector in the magnetic field direction, and $g_{B}=\boldsymbol{b} \cdot \boldsymbol{g}$ is the projection of the gravity acceleration on the magnetic field direction. Supplemented with the relation between $p$ and $\rho$, e.g. $p=$ const $\times \rho$ for an isothermal atmosphere, this equation determines the density variation along the magnetic field lines. The component of (1) perpendicular to the magnetic field reads

$(\nabla p)_{\perp}-\frac{1}{\mu_{0}}(\nabla \times \boldsymbol{B}) \times \boldsymbol{B}=\boldsymbol{g}_{\perp} \rho$,

where the perpendicular component of any vector $f$ is defined as $f_{\perp}=f-b(b \cdot f)$.

In a low-beta plasma, like in the solar corona, we can neglect the perpendicular gradient of plasma pressure, $(\nabla p)_{\perp}$, in comparison with the second term on the right hand side of Eq. (3). In coronal loops the characteristic scale of magnetic field variation in the perpendicular direction is the loop radius $a \sim 1 \mathrm{Mm}$. Taking $B \sim 30 \mathrm{G}$ we obtain that the second term on the left-hand side of Eq. (3) is of the order $10^{-3} \mathrm{~kg} \mathrm{~m}^{-2} \mathrm{~s}^{-2}$. On the other hand, since $g \approx 274 \mathrm{~m} \mathrm{~s}^{-2}$ and the typical value of the density in a coronal loop is $\rho=10^{-11} \mathrm{~kg} \mathrm{~m}^{-3}$, we obtain $\boldsymbol{g}_{\perp} \rho \sim 3 \times 10^{-8} \mathrm{~kg} \mathrm{~m}^{-2} \mathrm{~s}^{-2}$. These estimates imply that the righthand side of Eq. (3) can be neglected in comparison with the second term on its left-hand side, so that, with a very good accuracy, we can consider magnetic field in the solar corona as force-free, i.e. $(\nabla \times \boldsymbol{B}) \times \boldsymbol{B}=0$. Hence, when considering equilibrium configurations in the solar corona, we can choose the dependence of density in the directions perpendicular to the magnetic field arbitrarily. The equilibrium conditions only determine the density variation along the magnetic field lines.

In particular, we can consider equilibrium configurations with the density having a jump across definite magnetic surfaces. Of course, in the reality, the density is a continuous function of spatial coordinates. However, if we allow continuous density variation in the radial direction, then it will cause resonant damping of coronal loop oscillations (e.g., Ruderman \& Roberts 2002; Dymova \& Ruderman 2006). In order to get read of this effect we consider in this paper an idealized piece-wise constant in the radial direction density profile.

It has been shown by Dymova \& Ruderman (2005) that, in the first order approximation with respect to the small parameter $\epsilon=a / L$, the squared frequencies of the kink tube oscillations are the eigenvalues of the Sturm-Liouville problem

$\frac{\mathrm{d} Q}{\mathrm{~d} z^{2}}+\frac{\omega^{2}}{C_{\mathrm{k}}^{2}(z)} Q=0, \quad Q=0 \quad$ at $\quad z= \pm L$,

where

$Q=\frac{A}{\rho_{\mathrm{i}}-\rho_{\mathrm{e}}}, \quad C_{\mathrm{k}}^{2}=\frac{2 B^{2}}{\mu_{0}\left(\rho_{\mathrm{i}}+\rho_{\mathrm{e}}\right)}$.

Here $A(z)$ is the magnetic pressure perturbation at the tube boundary and $C_{\mathrm{k}}^{2}$ is the square of the kink speed of the tube which, in a stratified tube, is a function of $z$.

In the absence of longitudinal stratification, when $\rho_{\mathrm{i}}$ and $\rho_{\mathrm{e}}$ are constants, problem (4) has a well-known solution

$\omega_{m}^{2}=\left(\frac{\pi m}{L}\right)^{2} \frac{2 B^{2}}{\mu_{0}\left(\rho_{\mathrm{i}}+\rho_{\mathrm{e}}\right)}$, 


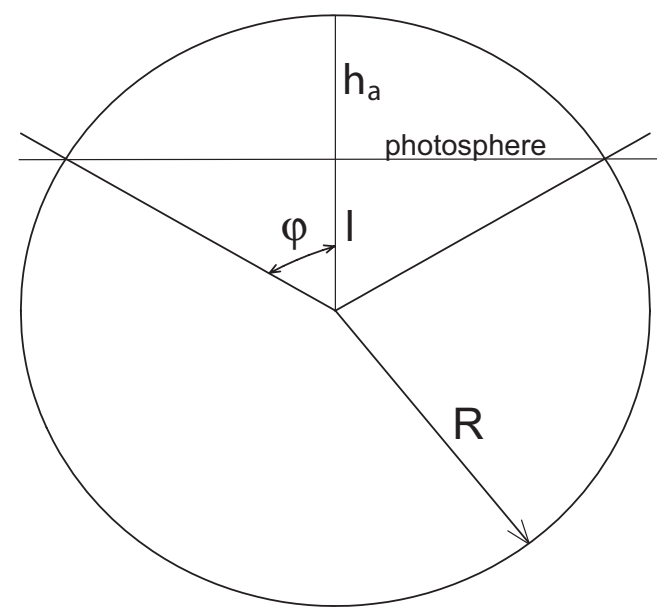

Fig. 2. The shape of a loop is an arc of a circle of radius $R$. The centre of the circle is at distance $l$ form the photosphere, $l$ being positive when the centre is below the photosphere and negative otherwise; $h_{\mathrm{a}}$ is the loop height.

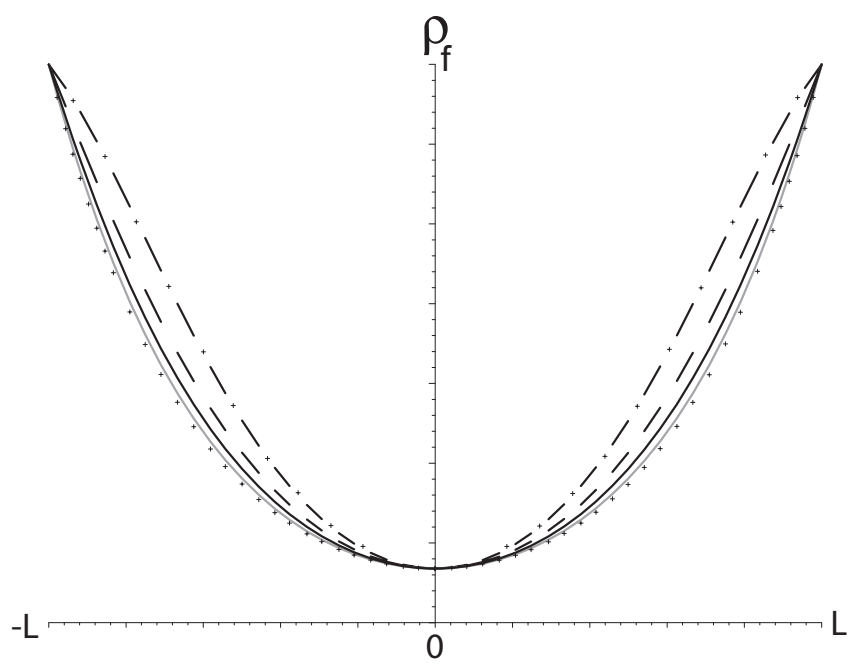

Fig. 3. Longitudinal density profiles given by expression (8) with $h_{\mathrm{a}}=$ $H$. Solid black, solid grey, dotted, dashed and dash-dotted lines correspond to the values of geometrical parameter $\lambda=0,0.5,0.9,-0.5,-0.9$ respectively.

where $m$ is a number of mode. It is clear that the ratio of the periods of the fundamental mode, $m=1$, and the first overtone, $m=2$, is equal to 2 in the unstratified case.

Since our equilibrium configuration allows arbitrary equilibrium density profile we consider different density profiles (Fig. 3) to calculate the frequencies of the kink mode of tube oscillations and compare the results. Let us consider an exponentially stratified atmosphere, i.e.

$\rho(h)=\rho_{\mathrm{f}} \exp (-h / H)$,

where $h$ is the height in the solar atmosphere, $H$ is the density scale height and $\rho_{\mathrm{f}}$ is the density at the photospheric level. We consider a loop in the form of an arc of a circle of radius $R$ (Fig. 2). The centre of the circle is at distance $l$ from the photosphere, $l$ being positive when the centre is below the photosphere and negative otherwise; $l=0$ corresponds to

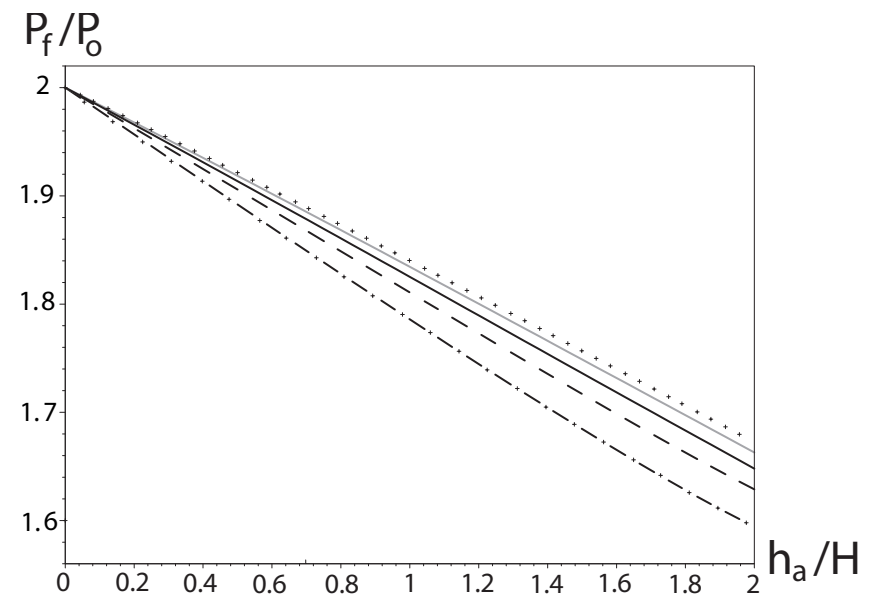

Fig. 4. The ratio of periods, $P_{\mathrm{f}} / P_{\mathrm{o}}$, of the fundamental kink mode and its first overtone as a function of the density stratification. Solid black, solid grey, dotted, dashed and dash-dotted lines correspond to the values of geometrical parameter $\lambda=0,0.5,0.9,-0.5,-0.9$ respectively.

a semicircular loop. The stratification function (7) projected on this curved loop yields the following density profile,

$\rho_{\mathrm{i}}(z)=\rho_{\mathrm{f}} \exp \left(-\frac{h_{\mathrm{a}}}{H} \frac{\cos (\zeta \varphi)-\lambda}{1-\lambda}\right)$,

where $\lambda=l / R, \varphi=\arccos \lambda, \zeta=z / L$ and $h_{\mathrm{a}}$ is the height of the loop apex point. The density profiles given by (8) are shown in Fig. 3 for different values of $\lambda$.

Substituting (8) into the Sturm-Liouville problem (4) we obtain

$$
\begin{array}{r}
\frac{\mathrm{d}^{2} Q}{\mathrm{~d} \zeta^{2}}+\Omega^{2} \exp \left(-\frac{h_{\mathrm{a}}}{H} \frac{\cos (\zeta \varphi)-\lambda}{1-\lambda}\right) Q=0, \\
Q=0 \quad \text { at } \zeta= \pm 1
\end{array}
$$

where $\Omega^{2}=\frac{\omega^{2} L^{2}}{C_{\mathrm{k} f}^{2}}$, with $C_{\mathrm{k} f}^{2}=\frac{2 B^{2}}{\mu_{0} \rho_{\mathrm{f}}(1+\chi)}$ being the kink speed at the footpoints.

The relative height of the loop as compared with the density scale height, $h_{\mathrm{a}} / H=L(1-\lambda) /(\varphi H)$, is used as a measure of stratification. Figure 4 shows the ratios of the period of the fundamental mode of the tube kink oscillations to its first overtone period, $P_{\mathrm{f}} / P_{\mathrm{o}}=\Omega_{\mathrm{o}} / \Omega_{\mathrm{f}}$, versus stratification parameter $h_{\mathrm{a}} / H$ for different values of $\lambda$. In fact, this ratio only depends on the stratification and the loop shape, while it is independent of all other parameters of the model, such as density contrast between the loop and its environment $\chi$. Solid black, solid grey, dotted, dashed and dash-dotted lines correspond to the values of geometrical parameter $\lambda=0,0.5,0.9,-0.5,-0.9$ respectively. For $h_{\mathrm{a}} / H=0$ the frequency of the first overtone is exactly double that of the fundamental one, while the ratio of the frequencies decreases as $h_{\mathrm{a}} / H$ increases. The black solid line for $\lambda=0$ is in good agreement with Fig. $1 \mathrm{~b}$ from the numerical study by Andries et al. (2005a) for a semicircular loop in an exponentially stratified atmosphere. In particular for the loop $\mathrm{C}$ from Verwichte et al. (2004) with $P_{\mathrm{f}} / P_{\mathrm{o}}=1.81$ and height of $70 \mathrm{Mm}$ we obtain an estimate for the density scale height about $65 \mathrm{Mm}$, and for the loop D with $P_{\mathrm{f}} / P_{\mathrm{o}}=1.64$ and loop height of $73 \mathrm{Mm}$ the density scale height estimate is $36 \mathrm{Mm}$, which are the same as those obtained by Andries et al. (2005a). The approximate coronal density scale heights in $\mathrm{Mm}$ for two loops $\mathrm{C}$ 
Table 1. Approximate values of the density scale height $H$ in the corona are given in Mm for different values of the geometrical parameter $\lambda$.

\begin{tabular}{ccc}
\hline \hline$\lambda$ & Case C & Case D \\
\hline 0 & 65 & 36 \\
0.5 & 61 & 34 \\
0.9 & 59 & 33 \\
-0.5 & 70 & 38 \\
-0.9 & 79 & 42 \\
\hline
\end{tabular}

and D from Verwichte et al. (2004) are given in Table 1 for some values of the geometrical parameter $\lambda$. Positive values of $\lambda$ correspond to loops flattened to the photosphere, e.g. $\lambda=0.9$ corresponds to an almost straight loop. Negative values of $\lambda$, on the contrary, correspond to loops more stretched out into the corona. Unfortunately at present observational error bars for the values of $P_{\mathrm{f}} / P_{\mathrm{o}}$ are larger than the variation of this ratio due to the loop shape parameter. But, as the present study shows, even if the parameter $P_{\mathrm{f}} / P_{\mathrm{o}}$ could be measured with a sufficient precision we would still need to know the loop shape to determine coronal density scale height correctly.

\section{Conclusions}

Inspired by the recent simultaneous observations of multiple harmonic coronal loop oscillations we looked at the effect of the loop geometry on the important observational parameter, the ratio of the periods of the fundamental mode, $P_{\mathrm{f}}$, and the first overtone, $P_{\mathrm{o}}$. We considered loops in the form of an arc of a circle of radius $R$ with the centre at distance $l$ from the photosphere.
The shape of the loop is determined by the parameter $\lambda=l / R$. We calculated the dependence of $P_{\mathrm{f}} / P_{\mathrm{o}}$ on the ratio of the loop height to the atmospheric scale height, $h_{\mathrm{a}} / H$, for different values of $\lambda$. Our main conclusion is that $P_{\mathrm{f}} / P_{\mathrm{o}}$ depends not only on $h_{\mathrm{a}} / H$, but also on the loop shape determined by the parameter $\lambda$. Hence, in order to use the observed values of $P_{\mathrm{f}} / P_{\mathrm{o}}$ for determining the scale height $H$, we also need to know the loop shape.

Acknowledgements. M.D. acknowledges the support by the University of Sheffield Fellowship and by the Overseas Research Students Award (ORS).

\section{References}

Andries, J., Arregui, I., \& Goossens, M. 2005a, ApJ, 624, L57

Andries, J., Goossens, M., Hollweg, J. V., Arregui, I., \& Van Doorsselaere, T. 2005b, A\&A, 430, 1109

Arregui, I., Van Doorsselaere, T., Andries, J., Goossens, M., \& Kimpe, D. 2005, A\&A, 441, 361

Aschwanden, M. J., Fletcher, L., Schrijver, C. J., \& Alexander, D. 1999, ApJ, 520,880

Aschwanden, M. J., De Pontieu, B., Schrijver, C. J., \& Title, A. M. 2002, Sol. Phys., 206, 99

Dymova, M., \& Ruderman, M. S. 2005, Sol. Phys., 229, 79

Dymova, M., \& Ruderman M. S. 2006, A\&A, 457, 1059

Nakariakov, V. M., Ofman, L., DeLuca, E. E., Roberts, B., \& Davila, J. M. 1999, Science, 285,862

Roberts, B., Edwin, P. M., \& Benz, A. O. 1984, ApJ, 279, 857

Ruderman, M. S., \& Roberts, B. 2002, ApJ, 577, 475

Schrijver, C. J., Aschwanden, M. J., \& Title, A. M. 2002, Sol. Phys., 206, 69

Uchida, Y. 1970, PASJ, 22, 341

Van Doorsselaere, T., Debosscher, A., Andries, J., \& Poedts, S. 2004, A\&A, 424, 1065

Verwichte, E., Nakariakov, V. M., Ofman, L., \& DeLuca, E. E. 2004, Sol. Phys., 223,77 